\title{
Parentalidad positiva: un eje para la intervención social con menores
}

\author{
Elisa Esteban-Carbonell \\ Universidad de Zaragoza \\ estecar@unizar.es
}

\section{Enrique Carnicero-Hernández}

Universidad de Zaragoza

kikecar@gmail.com

\author{
Nuria del Olmo-Vicén \\ Universidad de Zaragoza \\ nolmov@unizar.es
}

Los cambios en la realidad familiar traen consigo, entre otras cuestiones, una dificultad cada vez más creciente en el desempeño de las funciones parentales. En este ámbito, los programas de parentalidad positiva se erigen como un recurso que aporta herramientas y habilidades para hacer frente a las necesidades de los hijos e hijas. Sin embargo, la abundancia y variedad de estos programas hacen necesaria una profunda revisión y clasificación. En este artículo se proporciona una revisión de la perspectiva de la parentalidad positiva con el objetivo de aportar una exploración inicial para los investigadores y las investigadoras sociales de esta materia de estudio. Para ello, en un primer apartado, se exponen los elementos constitutivos de la parentalidad positiva. Un segundo apartado presenta las diferentes herramientas que la Unión Europea ha desplegado respecto a la parentalidad positiva. Finalmente, se revisan los elementos clave que siguen los programas y acciones de parentalidad positiva, distinguiendo por su mayor calidad a aquellos que están basados en la evidencia.

\section{Palabras Clave:}

Parentalidad positiva, intervención, familias, programas parentales, menores.

\section{GAKO-HITZAK:}

Gurasotasun positiboa, esku-hartzea, familiak, guraso-programak, adingabeak. 


\section{Introducción}

El modelo patriarcal de parentalidad ha sido sustituido hoy en día por la coexistencia de una gran diversidad de formas familiares donde la autoridad parental ya no recae únicamente en el padre cabeza de familia (Rodrigo, Máiquez, Martín, y Rodríguez, 2015). Ser madres y padres no es solo conseguir que las hijas y los hijos acepten las normas y les obedezcan, sino "promover comportamientos prosociales, la capacidad de pensar y entender el mundo que les rodea y el despliegue de una creciente autonomía personal y social" (Rodrigo et al., 2015: 28). Las madres y los padres sienten que, respecto a generaciones anteriores, han perdido influencia, lo que conduce a un deterioro de las responsabilidades parentales o a una parentalidad rígida y coercitiva.

Al mismo tiempo, las familias reciben pocos apoyos (económicos y psicoeducativos) (Rodrigo et al., 2015). Los programas de parentalidad positiva constituyen un nuevo modo de responder a las necesidades actuales de las familias. Apoyar la parentalidad es una prioridad de los Estados miembros de la Unión Europea y en ese sentido, el Consejo de Europa ha promovido la Recomendación 19/2006 sobre políticas de apoyo a la parentalidad positiva, donde se concreta el tipo de apoyos, porque la obligación de prestar los recursos necesarios viene ya recogida en la Convención sobre los Derechos del Niño de las Naciones Unidas (CNUDN) adoptada en $1989^{1}$. Asimismo, la Convención ha redefinido el papel de las niñas y los niños en las familias y en la sociedad, así como la relación de las madres y los padres con sus hijas e hijos. Ahora son portadores de derechos que deben ser garantizados por los Estados parte (Daly, 2007).

Por todo ello, el objetivo principal de este artículo es proporcionar una revisión de la intervención familiar con el fin de aportar una exploración inicial para los investigadores sociales de esta materia de estudio. Con este objetivo, en la primera parte se hace una aproximación al término "parentalidad positiva", incidiendo en sus elementos constituyentes. En la segunda parte, se presenta una revisión de las medidas de apoyo parental elaboradas desde la Unión Europea, incidiendo tanto en los organismos creados ad hoc como en las disposiciones normativas aprobadas. En la tercera parte, se ofrece algunas características sobre los programas de parentalidad positiva, haciendo una mención especial a los programas basados en la evidencia.

\section{Parentalidad positiva}

Antes de ahondar sobre el concepto de parentalidad positiva y sus elementos diferenciadores, es oportuno definir la parentalidad. Aunque existen Unidos) múltiples definiciones, la aportada por el Comité de Ministros es la más extendida a nivel europeo. En este sentido, "la parentalidad designa el conjunto actual de relaciones y actividades en las que los padres [y madres] están implicados para cuidar y educar a sus hijos [e hijas]" (Daly, 2007: 17). Es decir, la parentalidad implica una serie de tareas (cuidados físicos, establecimiento de límites, entre otras), de actitudes (afecto, mirada positiva, etc.), y de cualidades de relación (seguridad y apego positivo) (Quinton, 2004). Y, aunque no existe un acuerdo universal sobre el conjunto de tareas a desempeñar por las madres y los padres, Peænik (2012: 25), a partir de las aportaciones de Campion hace una síntesis importante donde recoge los derechos y responsabilidades de la parentalidad:

- Aportar los cuidados de base, proteger y educar al niño [y niña] hasta una edad adecuada;

- Garantizar la seguridad del niño [y niña];

- Orientarlo y fijarle límites;

- Asegurarle la estabilidad;

- Asegurar las condiciones del desarrollo intelectual, afectivo y social del niño [y niña], en especial la transmisión de ciertas competencias aceptadas y el modelado de los comportamientos deseados;

- Respetar la ley y contribuir a la seguridad de la sociedad;

- Contribuir a la prosperidad económica de la nación.

A pesar de la no existencia de un acuerdo sobre el término, la CNUDN señala que la parentalidad se compone de cuatro elementos: a) educación (que responde a la necesidad del niño y de la niña de afecto, seguridad, pertenencia y unión segura); b) estructura (establecimiento de normas de comportamiento y modelos de rol ofrecidos por las madres y los padres); c) reconocimiento (necesidad de la niña y del niño de ser aceptado); y d) capacitación (combinación del sentido del control personal con la capacidad para afectar el comportamiento del otro) (Peænik, 2012).

Dicho de otro modo, "la parentalidad o marentalidad es una forma semántica de referirse a las capacidades prácticas que tienen las madres y los padres para cuidar, proteger y educar a sus hijos [e hijas], y asegurarles un desarrollo suficientemente sano" (Barudy y Dantagnan, 2010: 34).

En este sentido, existe una extensa literatura en torno al concepto de parentalidad y sus tipos; por ejemplo se pueden identificar investigaciones relativas a la parentalidad biológica y parentalidad social (Barudy y Dantagnan, 2010), parentalidad mínima, parentalidad parcial, parentalidad disfuncional y parentalidad tóxica (Barudy, 2007), adecuación parental insuficiente, adecuación parental mínima, adecuación parental óptima (Rodrigo, Máiquez, Martín y Rodríguez, 2015). 
Aterrizando en el concepto de parentalidad positiva, la Recomendación 19/2006 del Comité de Ministros del Consejo de Europa (página 3) la define como:

[...] el comportamiento de [las madres y] los padres fundamentado en el interés superior [de la niña y] del niño, que cuida, desarrolla sus capacidades, no es violento y ofrece reconocimiento y orientación, que incluye el establecimiento de límites que permitan el pleno desarrollo [de la niña y] del niño.

En este sentido, se asiste a un cambio en la concepción de la infancia en el sentido que esta ya no es objeto de protección por parte de la figura parental, sino que pasa a ser un "sujeto activo de derechos que las figuras parentales deben promover, respetar y proteger" (Rodrigo et al., 2015: 12).

Por otra parte, existen diferentes perspectivas que permiten explicar en qué medida madres y padres deben promover determinados aspectos a sus hijos e hijas. En primer lugar, perspectivas unidireccionales que se apoyan entre otras en la teoría del apego (Ainsworth, Blehar, Waters y Wall, 1978; Bowlby, 1998, 1999), la teoría del aprendizaje social (Bandura, 1977, 1990) y la teoría de los estilos parentales (Baumrind, 1971) que identifican únicamente una influencia de padres y madres sobre sus hijos e hijas respecto a la parentalidad. Por otro lado, las teorías de bidireccionalidad parental (Kuczynski, 2003), que consideran que tanto los factores de las madres y los padres influyen en las niñas y los niños como al revés. En este sentido, los/as hijos/as al ejercer una influencia sobre las madres y los padres no son vistos como receptores de las conductas beneficiosas o perjudiciales de la parentalidad, sino que son sujetos activos en las relaciones paterno-filiales (relaciones de las madres y los padres con sus hijos/as). Y, por último, las teorías multifactoriales permiten explicar la parentalidad añadiendo el componente contextual. Belsky (1984) identifica tres determinantes que influyen en la parentalidad: los recursos psicológicos y personales de las madres y los padres, las características de la niña o del niño y las fuentes estresoras y/o de apoyo del contexto.

Por tanto, siguiendo la perspectiva multifactorial, son tres elementos los que influyen en la calidad de la parentalidad y que por tanto constituyen los elementos de la parentalidad positiva: a) el contexto psicosocial; b) las necesidades evolutivo-educativas de las niñas y de los niños; y c) las competencias parentales (Barudy y Dantagnan, 2007; Rodrigo et al., 2008; Rodrigo et al., 2010; Sallés y Ger, 2011).

El contexto psicosocial hace referencia a condiciones beneficiosas o protectoras o, por el contrario, perjudiciales o de riesgo que pueden influir en el funcionamiento familiar. Mientras que los factores de riesgo aumentan las probabilidades de la aparición de determinadas conductas que perjudique a los/as menores, los factores de protección modifican de forma beneficiosa la aparición de ese riesgo. Estos factores de protección y de riesgo pueden aparecer en la familia, el grupo de iguales, la escuela y la comunidad.

Las necesidades evolutivo-educativas de los niños y niñas incluyen aspectos tales como complicaciones en el nacimiento, retraso del desarrollo, presencia de enfermedades crónicas y/o discapacidades, entre otras situaciones. Estas necesidades pueden agruparse en cuatro tipos, siguiendo las aportaciones de López (2008): a) de carácter físicobiológico; b) mentales y culturales; c) emocionales y afectivas; y d) de participación social.

Y, por último, están las competencias parentales que, basándonos en la clasificación conceptual de Sallés y Ger (2011) se dividen en “capacidades fundamentales" y "habilidades" parentales. Las primeras refieren a "los recursos emotivos, cognitivos y conductuales de los que los progenitores disponen y que les permiten vincularse correctamente a sus hijos", mientras que las segundas conciernen la "plasticidad [de los padres que] les permite dar respuestas adecuadas y, al mismo tiempo, adaptar estas respuestas a las diferentes etapas de desarrollo" (Sallés y Ger, 201: 48-49).

Se constata, por tanto, una amplia literatura sobre la parentalidad y, específicamente, sobre la parentalidad positiva, siendo clave el papel jugado por la Unión Europea, quien define el concepto e identifica la necesidad de procurar medios y recursos para apoyar a las familias al desarrollo óptimo de sus hijos/as. Hasta llegar a la Recomendación 19/2006 del Comité de Ministros del Consejo de Europa, es necesario conocer la evolución de las diferentes acciones promovidas en la Unión Europea que incorporan una nueva perspectiva sobre la que intervenir con la infancia.

\section{El apoyo a la familia desde la Unión Europea}

A lo largo de las últimas décadas han aparecido numerosas acciones europeas que inciden $\mathrm{y} / 0$ promueven una parentalidad positiva. En 2002, los Gobiernos europeos redactaron los objetivos de Barcelona para eliminar los frenos de la participación de las mujeres en el mercado de trabajo y crear estructuras de acogida para al menos el $90 \%$ de los/as niños/as entre tres años y la edad de escolaridad obligatoria, y para al menos el $33 \%$ de los niños/as de menos de tres años. En 2010 se adoptó una declaración ministerial apelando a la creación de servicios para la primera infancia mejor desarrollados y de mejor calidad (Daly y Martin, 2013).

Por otra parte, entre los principales medios utilizados por la Unión Europea, las plataformas permiten el debate y la difusión sobre aspectos concretos. En 1989, se puso en marcha un Observatorio Europeo 
de Políticas Familiares Nacionales, rebautizado Observatorio Europeo de Asuntos Sociales y, más recientemente, Observatorio Europeo de la Situación Social, la Demografía y la Familia. En la misma línea se encuentra la Alianza Europea para las Familias, establecida en marzo de 2007 , y que proporciona un espacio de debate e intercambio de información e iniciativas en el campo de la protección a la familia. También Eurochild, como red europea que agrupa a las ONG que trabajan en la promoción del bienestar en la infancia.

En 2007 el Consejo de Europa publica un informe donde propone una concepción de la parentalidad llamada "positiva" (positive parenting). Se concibe inicialmente en el marco de una reflexión para reducir la violencia sufrida por la infancia, interesándose sobre las condiciones sociales necesarias para que la función parental respete el interés superior de la infancia (Daly y Martin, 2013). Asimismo, en 2011 se llevó a cabo una estrategia coordinada en Europa sobre parentalidad positiva cuyo resultado fue la publicación de un informe que reúne iniciativas de diferentes países concluyendo en la necesidad de alinear esfuerzos en el campo de la parentalidad positiva con la estrategia Europa 2020 (Arranz y Rodrigo, 2018). Dicha estrategia confiere un papel relevante al apoyo parental, integrándose en dos de las diez directrices: reducción del abandono escolar (directriz 9) y reducción de los que viven bajo el umbral de pobreza en 20 millones para 2020 y promover la inclusión social (directriz 10).

Por su parte, la recomendación del Consejo de Europa refuerza esa nueva visión del proceso de socialización primaria donde tanto madres y padres como hijas e hijos tienen un papel activo en la conformación de la conducta a partir de la adquisición compartida de creencias, valores y normas (Peænik, 2012). La socialización ya no es responsabilidad única de la familia puesto que, de ser así, no se producirían procesos de cambio en los valores, en las creencias y en las normas. Este nuevo enfoque otorga "la máxima importancia a la contribución progresiva que tienen los hijos y las hijas al proceso de socialización, así como a la dialéctica de construcción y colaboración mutua que se va creando entre unos y otros" (Martín, Cabrera, León y Rodrigo, 2013: 79). El objetivo es reconocer oficialmente la responsabilidad parental y la necesidad de contar con apoyos en el desempeño parental (Consejo de Europa, 2006). Asumir la importancia de la parentalidad implica desarrollar una política familiar a través de: a) medidas de política generales, b) parentalidad positiva, c) servicios de apoyo a [las madres y a] los padres, d) servicios para [madres y] padres en riesgo de exclusión social y e) principios de orientación a los/ as profesionales.

A pesar de estas líneas comunes sobre la parentalidad positiva y las acciones o estrategias de apoyo parental, cada país ha destinado sus esfuerzos y recursos de distinta forma. En este sentido, resulta especialmente interesante el trabajo de Daly (2011), quien describe las políticas y experiencias de Francia, Bélgica, Bulgaria, Croacia, República Checa, Dinamarca, Alemania, Estonia e Italia.

Si bien el Consejo de Europa ya avanza tipos de programas o intervenciones destinadas a madres y a padres en función de distintos criterios (momento evolutivo, situación económica, presencia de diversidad funcional, etc.), no concreta contenidos y/o metodologías de intervención. No hay que olvidar, en este sentido, que los Estados son una más de las partes determinantes de la "comunidad", tal y como afirma Daly (2007). Esta autora señala que la parentalidad requiere medios y "capital", así como derechos y obligaciones para lograr tales medios. Estos derechos y obligaciones implican a diversas partes de la "comunidad" entre los que se encuentran los Estados, pero también las madres y los padres, las niñas y los niños y los proveedores de servicios locales y nacionales. Cada una de estas partes tiene su responsabilidad respecto a la parentalidad y debe contar con los medios necesarios para llevarla a cabo (Daly, 2007).

Se puede concluir que, a pesar de la falta explícita de concreción por parte del Consejo de Europa, sí se evidencia el interés en fomentar medidas que contribuyan a desarrollar políticas familiares cuyo eje principal establezca principios de parentalidad positiva; en este sentido en el siguiente apartado se revisan los elementos clave que siguen los programas y acciones de parentalidad positiva para ser considerados de calidad, haciendo mención especial a los procesos de evaluación, distinguiendo por su mayor calidad a aquellos que están basados en la evidencia.

\section{Algunos elementos clave de la calidad de los programas y acciones basados en la parentalidad positiva}

En este contexto, el concepto de programas de parentalidad positiva "se entenderá como el referido a un conjunto de actividades o servicios regulares y estructurados, dirigidos a familias con niños menores o durante la perinatalidad, con el fin de brindar apoyo y contribuir al desarrollo de competencias y habilidades parentales varias" (Esteban y Firbank, 2019: 100).

Nuevamente, nos encontramos ante un ámbito de investigación extenso que se ha esforzado por definir los diferentes tipos de programas y sus rasgos diferenciadores (Consejo de Europa, 2011; Martín, 2005; Martín-Quintana et al., 2009; Sandbaek, 2007; Vila, 1998), así como por describir la evolución temporal de estos (Máiquez et al., 2000; Martín, 2005; Martín-Quintana et al., 2009; Vila, 1998). Entre todos ellos, es interesante destacar las aportaciones de Arranz y Rodrigo (2018), quienes agrupan los programas en tres categorías. Por un lado, programas 
basados en evidencia científica, que generalmente usan una metodología constructivista e incluyen procesos de evaluación sistemática y procedimientos para incorporar mejoras. Por otro lado, acciones y programas individuales para familias dirigidos tanto a familias "en riesgo" como en "no riesgo", que han sido referidos por los servicios sociales, educativos o de salud. Algunos permiten una evaluación exhaustiva y otros tienen como objetivo difundir información y/o proporcionar orientación a las familias de acuerdo con los principios básicos de la parentalidad positiva. Y, por último, recursos para dar apoyo al trabajo de los profesionales con las familias, que incluyen formación online y presencial a través de cursos sobre diseño de programas, estrategias de evaluación, etc.

Por tanto, encontramos diversas experiencias sobre programas de parentalidad positiva y de ello dan cuenta las numerosas revisiones sistemáticas y estudios realizados al respecto (Altafim y Linhares, 2016; Capano-Bosch, González-Tornaría, Navarrete y Mels, 2018; Morrison, Pikhart, Ruiz y Goldblatt, 2014; Rodrigo, 2016; Rubio, Trillo y Jiménez, 2020; Vaquero, Suárez, Fernández, Rodrigo y Balsells, 2019). Sin embargo, no todos los programas o acciones son prácticas basadas en la evidencia, dado que, o no han sido sometidas a una evaluación científica, 0 sus resultados no han sido los adecuados (Rodrigo, Almeida, Spiel y Koops, 2012).

Mostrar la evidencia científica y, por consiguiente, la efectividad de los programas es un punto clave para dotar de validez y fiabilidad a este tipo de intervenciones, al mismo tiempo que contribuyen al desarrollo de un sector específico de políticas sociales. En esta línea, las investigaciones recientes tratan de definir los criterios que deben seguir los programas para ser considerados de calidad (Jiménez e Hidalgo, 2016). Molinuevo (2013), entre otros, señala que cualquier programa debe incluir un diagnóstico del contexto (necesidades y recursos, entre otros), un marco teórico o modelo explicativo (Hidalgo et al., 2012), y un sistema de evaluación que permita valorar la eficacia, la efectividad y la eficiencia de las intervenciones (Máiquez et al., 2015).

La eficacia comprueba si el programa ha producido los cambios esperados en relación con objetivos, metodología y contenidos, en condiciones ideales de implementación. Para la efectividad se evalúan los aspectos anteriores, junto al nivel de abandono del programa en condiciones de la vida real. Y la eficiencia de un programa sirve para identificar si es posible generalizar resultados y optimizar ciertos aspectos con el fin de mejorar el programa y su aplicabilidad (Máiquez et al., 2015). Junto a estos tres tipos de evaluación, es necesario responder a cómo se evalúa, qué se evalúa y quién evalúa. Por su parte, Jiménez e Hidalgo (2016) muestran tres tipos de evaluación (de eficacia, de eficiencia y de diseminación) que constituyen niveles diferenciados dentro de un todo, por lo que para que un tipo de evaluación se lleve a cabo deben haberse completado la anterior.

La evaluación es un aspecto crucial y muestra de ello es el extenso repertorio de evaluaciones de programas (Marinho y Ferreira, 2000; Martín, Máiquez, Rodrigo, Correa y Rodríguez, 2004; Gómez, Cifuentes y Ortún, 2012; Amorós, Balsells, Buisan, Byrne, y Fuentes-Peláez, 2013; Álvarez, Padilla, Byrne, Máiquez y Rodrigo, 2015; Hidalgo et al., 2015; Martínez, Pérez, Álvarez, Rodríguez y Becedóniz, 2015; Mendes, Mattar y Almeida, 2015; Muñoz y Gómez, 2015; Rodríguez, Martín y Rodrigo, 2015; Tenorio, Garavito, Sánchez y Burbano, 2015; Torres et al., 2015; Ramos, Vázquez, Pasarín y Artazcoz, 2016; Torío, Fernández e Inda, 2016); sin embargo, todavía hay escasos estudios sobre la implementación y evaluación en la primera infancia (Ferrer y Riera, 2014).

Paralelamente, conviene señalar que existen diferentes posiciones respecto a los procedimientos para llevar a cabo la evaluación. Inicialmente se ha utilizado la perspectiva de corte experimental con grupos de control de asignación. Posteriormente, otra corriente que apuesta por una visión más global de la evaluación, donde la metodología importa, pero también la utilidad, la viabilidad y el rigor ético (Jiménez e Hidalgo, 2016).

En cualquier caso, a pesar de que se cuente con evidencias de que un programa es eficaz, puede tener resultados negativos a la hora de implementarse por la influencia en el éxito o en el fracaso de la intervención de ciertos aspectos, como el apoyo institucional, la formación de los y las profesionales, la flexibilidad y la fidelidad del programa (Jiménez e Hidalgo, 2016). El apoyo institucional es fundamental, pero también se deben garantizar unos recursos apropiados, lo que implica una formación específica de los y las profesionales. Por otra parte, es preciso encontrar el equilibrio entre la fidelidad al programa original (hay un mayor éxito cuanto más se asemeja a la esencia del programa), y la flexibilidad en su adaptación a otros contextos sin desvirtuar el programa. Hay que conocer qué aspectos son susceptibles de ser modificados y cuáles son inamovibles (Jiménez e Hidalgo, 2016).

Por todo ello, una buena práctica es aquella experiencia con principios, objetivos y procedimientos adecuados, que ha tenido resultados positivos, probando su eficacia y utilidad en un contexto determinado. Se distinguen tres niveles de buenas prácticas (Rodrigo et al., 2015):

1. Práctica profesional individualizada: basada en la propia experiencia profesional u observación directa de las prácticas de otros/as profesionales. Un saber subjetivo que no se difunde.

2. Práctica profesional consensuada: el saber se comparte entre profesionales y se llega al consenso sobre las "buenas prácticas", las cuales identifican, describen y difunden para convertirse en un conocimiento compartido. 
3. Práctica basada en evidencias: los resultados de la investigación se difunden y divulgan para que constituyan una práctica informada.

Por último, este tipo de programas cuenta con una descripción detallada de la intervención y de la evaluación para facilitar ser replicado. El manual debe incluir la población objetivo, los mecanismos por los cuales la intervención debería funcionar, una descripción detallada de su contenido y organización, procedimientos de intervención, materiales, pautas de evaluación, etc. (Jiménez, Antolín-Suárez, Lorence e Hidalgo, 2018).

\section{Conclusiones}

A través de estas páginas se ha reflexionado sobre la parentalidad positiva y, específicamente, sobre los programas que incorporan dicha perspectiva. La proliferación de acciones y programas destinados a las familias cuyo objetivo es la mejora de las prácticas parentales de crianza de niños/as tiene un largo recorrido, en el que las instituciones europeas han jugado un papel clave gracias a las distintas medidas adoptadas. A partir de ellas, la infancia se sitúa en una posición destacada, donde su voz cuenta.

La conceptualización de la parentalidad positiva permite conocer mejor sus rasgos diferenciadores para identificar sobre qué aspectos se puede trabajar desde el ámbito de la intervención. Las teorías multifactoriales a través de las cuales se explica la parentalidad permiten conocer qué aspectos influyen en ella. Es decir, el contexto se erige como un elemento que influye en la parentalidad. Al mismo tiempo, se han mostrado los aspectos teóricos que permiten identificar a los programas basados en la evidencia como programas de calidad, diferenciándolos de las buenas prácticas. Un buen programa debe contar con un buen diseño que incorpore todos los elementos necesarios para implementarse, pero también para poder ser evaluado. Los programas basados en la evidencia tienen que poder ser replicados en otros contextos, guardando la esencia del programa, aunque adaptándolo al contexto concreto, lo cual garantiza unos criterios de calidad o éxito del programa. 
AINSWORTH, M.D.S.; BLEHAR, M.C.; WATERS, E. y WALL, S. (1978): Patterns of Attachment, Hillsdale, Erlbaum.

ALTAFIM, E.R.P. y LINHARES, M.B.M. (2016): “Universal violence and child maltreatment prevention programs for parents: A systematic review", Psychosocial Intervention. Psychosocial Intervention, vol. 25, n- 1 , pp. 27-38, 〈https://doi.org/10.1016/j. psi.2015.10.003).

ÁLVAREZ, M.; PADILLA, S.; BYRNE, S.; MÁIQUEZ, M.L. y RODRIGO, M.J. (2015): “Crecer felices en familia: un programa de apoyo parental para el desarrollo infantil”, En M. RODRIGO, M. MÁIQUEZ, J. MARTÍN, S. BYRNE, y B. RODRÍGUEZ, Manual práctico de parentalidad positiva, Madrid, Síntesis, pp. 91-110.

AMORÓS, P.; BALSELLS, M.; BUISAN, M.; BYRNE, S. y FUENTES-PELÁEZ, N. (2013): “Implementation and evaluation of the 'Learning together, growing in family programme': The impact on the families", Revista de cercetare si interventie sociala, no 42, pp. 120-144.

ARRANZ, E. y RODRIGO, M. (2018): "Positive parenting in Spain: introduction to the special issue", Early child development and care, vol. 188, n- $11, \mathrm{pp}$. 1503-1513, 〈https://doi.org/10.1080/03004430 .2018.1501565>

BANDURA, A. (1977): Social Learning Theory, Nueva York, General Learning Press.

- (1990): Aprendizaje social y desarrollo de la personalidad, Madrid, Alianza.

BARUDY, J. (2007): "Familiaridad y competencias: el desafío de ser padres", en BARUDY, J. y DANTAGNAN, $M .$, Los buenos tratos a la infancia. Parentalidad, apego y resiliencia, Barcelona, Gedisa, págs. 77-125.
BARUDY, J. y DANTAGNAN, M. (2007): Los buenos tratos a la infancia. Parentalidad, apego y resiliencia, Barcelona, Gedisa.

- (2010): Los desafíos invisibles de ser madre o padre. Manual de evaluación de las competencias y la resiliencia parental, Barcelona, Gedisa.

BAUMRIND, D. (1971): “Current patterns of parental authority”, Developmental Psychology, vol. 4, nํㅜ 1, págs. 1-103.

BOWLBY, J. (1998): El apego y la pérdida. Vol. 1. El apego, Barcelona, Paidós.

- (1999): Vínculos afectivos: formación, desarrollo y pérdida, Madrid, Morata.

BELSKY, J. (1984): "The determinants of parenting: A process model”, Child Development, vol. 55, $\mathrm{n}$ 을, págs. 83-96.

CAPANO-BOSCH, A.; GONZÁLEZ-TORNARÍA, M.L.; NAVARRETE, I. y MELS, C. (2018): “Del castigo físico a la parentalidad positiva. Revisión de programas de apoyo parental", Revista de Psicología, vol. 14, no 27, pp. 125-138, recuperado de: $\langle$ http://bibliotecadigital. uca.edu.ar/greenstone/cgi-bin/library. cgi $? \mathrm{a}=\mathrm{d} \& \mathrm{c}=$ Revistas $\& \mathrm{~d}=$ castigo - fisico parentalidadpositiva〉.

CONSEJO DE EUROPA (2006): Recommendation Rec (2006)19 of the Committee of Ministers to member states on policy to support positive parenting.

- (2011): Recommendation Rec (2011)12 of the Committee of Ministers to member states on children's rights and social services friendly to children and families.

DALY, M. (2007): La parentalidad en la Europa contemporánea: un enfoque positivo. Consejo de Europa. 
- (2011): Building a coordinated strategy for parenting support, European Commision Employement, Social Affairs and Inclusion.

DALY, M. y MARTIN, C. (2013): “Le soutien à la parentalité”, Informations sociales, vol. 175, nㅇ 1, pp. 120128, 〈https://doi.org/10.3917/inso.175.0120〉.

ESTEBAN, E. y FIRBANK, O. (2019): “Parentalidad positiva, riesgo e intervención: un análisis de los dispositivos de apoyo vigentes en Québec", Cuadernos de Trabajo Social, Vol. 32, nำ 1, pp. 99-111, 〈https://doi.org/10.5209/CUTS.56715〉.

FERRER, M. y RIERA, M. (2014): “Observación y evaluación de los profesionales en los programas de apoyo a la parentalidad en la primera infancia: proceso de elaboración de un instrumento", Cuadernos de Trabajo Social, vol. 27, $n \div 0$ 2, pp. 405-415, <https://doi.org/10.5209/rev_ CUTS.2014.v27.n2.44510〉.

GÓMEZ E.; CIFUENTES, B. y ORTÚN, C. (2012): "Padres Competentes, Hijos Protegidos: Evaluación de Resultados del Programa 'Viviendo en Familia”, Psychosocial Intervention, Vol. 21, n은 3, pp. 259-271.

HIDALGO, M.V.; MENÉNDEZ, S.; LÓPEZ-VERDUGO, I.; SÁNCHEZ, J.; LORENCE, B. y JIMÉNEZ, L. (2015): "Programa de formación y apoyo familiar", en M. RODRIGO, M. MÁIQUEZ, J. MARTÍN, S. BYRNE, y B. RODRÍGUEZ, Manual práctico de parentalidad positiva, Madrid, Síntesis, pp. 169-188.

HIDALGO, M.V.; OLIVA, A.; JIMÉNEZ, L.; ANTOLÍN-SUÁREZ, L.; MENÉNDEZ, S. y LORENCE, B. (2012): Proyecto de Apoyo Integral a las Familias (PAIF), Sevilla, Universidad de Sevilla y Gobierno de Cantabria.

JIMÉNEZ, L. e HIDALGO, M.V. (2016): “La incorporación de prácticas basadas en evidencias en el trabajo con familias: los programas de promoción de parentalidad positiva", Apuntes de Psicología, vol. 34, nํ- 2-3, pp. 91-100.

JIMÉNEZ, L.; ANTOLÍN-SUÁREZ, L.; LORENCE, B. e HIDALGO, V. (2018): "Family education and support for families at psychosocial risk in Europe: Evidence from a survey of international experts", Health \& Social Care in the Community, vol. 27, nํㅜ 2, pp. 1-10, <https://doi. org/10.1111/hsc.12665'.

KUCZYNSKI, L. (2003): “Beyond bidirectionality: Bilateral conceptual frameworks for understanding dynamics in parent-child relations", en KUCZYNSKI, L. (ed.), Handbook of Dynamics in Parent-Child Relations, Thousand Oaks, Sage, págs. 1-24.

MÁIQUEZ, M.; RODRIGO, M. y BYRNE, S. (2015): “El proceso de apoyo en la promoción de la parentalidad positiva”, en M. RODRIGO, M. MÁIQUEZ, J. MARTÍN, S. BYRNE, y B. RODRÍGUEZ: Manual práctico de parentalidad positiva, pp. 67-87, Madrid, Síntesis.

MÁIQUEZ, M.; RODRIGO, M.; CAPOTE, C. y VERMAES, I. (2000): Aprender en la vida cotidiana. Un programa experiencial para padres, Madrid, Visor.

MARINHO, M. y FERREIRA, E. (2000): “Evaluación de la eficacia de un programa de entrenamiento de padres en grupo", Psicología Conductual, vol. 8, nํㅜ 2, pp. 299-318.
MARTÍN, J.C. (2005): “Evaluación del programa de apoyo personal y familiar para familias en situación de riesgo psicosocial" [tesis doctorial], Universidad de La Laguna.

MARTÍN, J., CABRERA, E., LEÓN, J., y RODRIGO, M. (2013): “La Escala de Competencia y Resiliencia Parental para madres y padres en contextos de riesgo psicosocial”, Anales de Psicología, vol. 29, nํㅜ 3, pp. 886-896.

MARTÍN, J.C.; MÁIQUEZ, M.L.; RODRIGO, M.J.; CORREA, A.D. y RODRÍGUEZ, G. (2004): “Evaluación del programa 'Apoyo personal y familiar' para madres y padres en situación de riesgo psicosocial", Infancia y Aprendizaje, vol. 27, n 4 , pp. 437-445.

MARTÍN-QUINTANA, J.C.; BYRNE, S.; MÁIQUEZ, M.L.; RODRÍGUEZ, B.; RODRIGO, M. J. y RODRÍGUEZ, G. (2009): "Programas de Educación Parental”, Intervención Psicosocial, vol. 18, nํㅜ 2, pp. 121-133.

MARTÍNEZ, R.A.; PÉREZ, M.H.; ÁLVAREZ, L.; RODRÍGUEZ, B. y BECEDÓNIZ, C. (2015): "Programa basado en evidencias para fomentar la parentalidad positiva en Asturias (España)", en M. RODRIGO LÓPEZ, M. MÁIQUEZ CHAVES, J. MARTÍN QUINTANA, S. BYRNE, y B. RODRÍGUEZ RUÍZ, Manual práctico de parentalidad positiva, Madrid, Síntesis, pp. 151-168.

MENDES, N.; MATTAR, M.A. y ALMEIDA, A.T. (2015): "Educación parental en familias en situación de pobreza en el contexto brasileño”, en M. RODRIGO, M. MÁIQUEZ, J. MARTÍN, S. BYRNE, y B. RODRÍGUEZ, Manual práctico de parentalidad positiva, Madrid, Síntesis, pp. 223-244.

MOLINUEVO, D. (2013): Parenting support in Europe, Dublín, Eurofound.

MORRISON, J.; PIKHART, H.; RUIZ, M. y GOLDBLATT, P. (2014): "Systematic review of parenting interventions in European countries aiming to reduce social inequalities in children's health and development", BMC. Public Health, vol. 14 , pp. 1-13, recuperado de: 〈https:// bmcpublichealth.biomedcentral.com/ articles/10.1186/1471-2458-14-1040>.

MUÑOZ, M.M. y GÓMEZ, E.A. (2015): “Crecer en familia: un servicio para promover la parentalidad positiva en Chile”, en M. RODRIGO, M. MÁIQUEZ, J. MARTÍN, S. BYRNE, y B. RODRÍGUEZ, Manual práctico de parentalidad positiva, Madrid, Síntesis, pp. 189-204.

PEAENIK, N. (2012): “Hacia una visión de la parentalidad en el interés superior del niño”, en M. DALY, La parentalidad en la Europa contemporánea: un enfoque positivo, Consejo de Europa, pp. 21-46.

QUINTON, D. (2004): Supporting parents. Messages from research, London, Jessica Kingsley.

RAMOS, P.; VÁZQUEZ, N.; PASARÍN, M. y ARTAZCOZ, L. (2016): “Evaluación de un programa piloto promotor de habilidades parentales desde una perspectiva de salud pública", Gaceta Sanitaria, vol. 30, nำ 1, pp. 37-42, 〈https://doi. org/10.1016/j.gaceta.2015.08.008〉.

RODRIGO, M.J. (2016): “Quality of implementation in evidence-based positive parenting programs in Spain: Introduction to the special issue", 
Psychosocial Intervention, vol. 25, no 2, pp. 63-68, «https://doi.org/10.1016/j.

psi.2016.02.004>.

RODRIGO, M.J.; ALMEIDA, A.; SPIEL, C. y KOOPS, W. (2012): "Introduction: Evidence-based parent education programmes to promote positive parenting", European Journal of Developmental Psychology, vol. 9, no- 1, pp. 2-10, 〈https://doi. org/10.1080/17405629.2011.631282>.

RODRIGO, M.; MÁIQUEZ, M.; MARTÍN, J. y BYRNE, S. (2008): Preservación familiar: un enfoque positivo para la intervención con familias, Madrid, Pirámide.

RODRIGO, M.; MÁIQUEZ, M.; MARTÍN, J. y RODRÍGUEZ, B. (2015): "La parentalidad positiva desde la prevención y la promoción”, en M. RODRIGO, M. MÁIQUEZ, J. MARTÍN, S. BYRNE, y B. RODRÍGUEZ, Manual práctico de parentalidad positiva, Madrid, Síntesis, pp. 25-43.

RODRÍGUEZ, E.; MARTÍN, J.C. y RODRIGO, M.J. (2015): “Vivir la adolescencia en familia. Un programa de apoyo parental en la adolescencia", en M. RODRIGO, M. MÁIQUEZ, J. MARTÍN, S. BYRNE, y B. RODRÍGUEZ, Manual práctico de parentalidad positiva, Madrid, Síntesis, pp. 131-150.

RUBIO, F.J.; TRILLO, M.P. y JIMÉNEZ, M.C. (2020): “Programas grupales de parentalidad positiva: una revisión sistemática de la producción científica”, Revista de Educación, vol. 24, no 389, págs. 267-295, 〈https://doi.org/10.4438/1988-592XRE-2020-389-462>.

SALLÉS, C. y GER, S. (2011): “Las competencias parentales en la familia contemporánea: descripción, promoción y evaluación”, Educación Social, vol. 49, págs. $25-47$.

SANDBAEK, M. (2007): "Servicios de apoyo a una parentalidad positiva”, En M. DALY, La parentalidad en la Europa contemporánea: un enfoque positivo, Consejo de Europa, pp. 71-102.

TENORIO, M.C.; GARAVITO, J.; SÁNCHEZ, J.E. y BURBANO, M.H. (2015): "Familia y escuela educamos juntos”, en M. RODRIGO, M. MÁIQUEZ, J. MARTÍN, S. BYRNE, y B. RODRÍGUEZ, Manual práctico de parentalidad positiva, Madrid, Síntesis, pp. 205-222.

TORÍO, S.; FERNÁNDEZ, C.M. e INDA, M.M. (2016): "Evaluación de un programa experiencial de educación parental: la perspectiva de los educadores como agentes de cambio", Aula Abierta, vol. 44, pp.31-37, 〈https://doi. org/10.1016/j.aula.2015.05.001>.

TORRES, A.; SUÁREZ, A.; ÁLVAREZ, M.; PADILLA, S.; RODRÍGUEZ, E. y RODRIGO, M.J. (2015): “Apoyo parental 'online”, en M. RODRIGO, M. MÁIQUEZ, J. MARTÍN, S. BYRNE, y B. RODRÍGUEZ, Manual práctico de parentalidad positiva, Madrid, Síntesis, pp. 245-263.

VAQUERO, E.; SUÁREZ, A.; FERNÁNDEZ, L.; RODRIGO, M.J. y BALSELLS, M.A. (2019): “E-parenting: una revisión sistemática de la literatura”, EDUTEC. Revista Electrónica de Tecnología Educativa, vol. 68, pp. 30-41, 〈https://doi.org/10.21556/ edutec.2019.68.1313〉.

VILA, I. (1998): “Intervención psicopedagógica en el contexto familiar”, en M. RODRIGO, y J. PALACIOS, Familia y desarrollo humano, Madrid, Alianza Editorial, pp. 501-519.

\section{Agradecimientos}

Parte de este trabajo se ha realizado con la colaboración del Departamento de Innovación, Investigación y Universidad del Gobierno de Aragón. 
